(c) Elsevier/INRA

\title{
Influence du froid, du vent et de la pluie sur les dépenses énergétiques et la thermorégulation de sept types génétiques de brebis
}

\author{
JF Hocquette ${ }^{1}, \mathrm{M}_{\text {Vermorel }}{ }^{1 *}$, J Bouix ${ }^{2}$ \\ avec la collaboration technique de Y Anglaret, JP Donnat, \\ C Leoty, M Meyer, R Souchet \\ ${ }^{1}$ Institut national de la recherche agronomique, laboratoire croissance \\ et métabolisme des herbivores, centre de recherches de Clermont-Ferrand/Theix, \\ 63122 St Genès-Champanelle; \\ ${ }^{2}$ Institut national de la recherche agronomique, station d'amélioration \\ génétique des animaux, centre de recherches de Toulouse, \\ 31326 Castanet-Tolosan, France
}

(Reçu le 5 octobre 1990; accepté le 30 janvier 1992)

\begin{abstract}
Résumé - La production de la chaleur de 64 brebis de 7 types génétiques d'ovins a été mesurée en chambres respiratoires pour déterminer l'influence sur leurs dépenses énergétiques, de conditions climatiques bien contrôlées simulant les intempéries rencontrées en alpage. $\grave{A} 15^{\circ} \mathrm{C}$, en air calme, la thermogenèse des brebis est en moyenne de $3,55 \mathrm{kcal} / \mathrm{kg}$ $P^{0,75}$ par h. Une réduction de $5^{\circ} \mathrm{C}$ de la température ambiante ou un vent turbulent de 2 à $4 \mathrm{~m} / \mathrm{s}$ entraîne une augmentation de $9 \%$ de la thermogenèse. Exposées à la pluie et au vent, les brebis s'immobilisent, la tête basse et le dos au vent. La thermogenèse des brebis Préalpes et Mourérous augmente rapidement (en 1,5 h) et fortement $(+101 \%$ et $+120 \%$ respectivement) et, dans une moindre mesure, celle des brebis Communes des Alpes $(+71 \%)$. Ces races semblent cependant s'accoutumer assez rapidement aux intempéries car l'augmentation de leur thermogenèse n'est plus que de l'ordre de $40 \%$ le second jour. La thermogenèse des types génétiques Mérinos et apparentés au Mérinos («mérinisés») augmente plus lentement (en 3 à $4 \mathrm{~h}$ ) et plus faiblement $($ de +22 à $+78 \%)$ avec les intempéries sans réduction le deuxième jour, excepté pour les brebis $F_{1}$ Romanov $\times$ Mérinos d'Arles. Les brebis de souche Mérinos ont une toison très étendue, couvrant le ventre et très isolante car épaisse et dense. Les concentrations plasmatiques de triiodothyronine $\left(\mathrm{T}_{3}\right)$,
\end{abstract}

* Correspondance et tirés à part : M Vermorel, INRA, Theix, 63122 Saint-Genès Champanelle 
thyroxine $\left(\mathrm{T}_{4}\right)$, glucose et acides gras non estérifiés augmentent de $26,15,8$ et $56 \%$ respectivement après $2 \mathrm{j}$ d'exposition aux intempéries. Les types génétiques les plus sensibles aux intempéries sont les Préalpes et les Mourérous, suivis des Communes des Alpes. Les types génétiques «mérinisés» apparaissent mieux adaptés aux intempéries, spécialement les races Est à laine Mérinos et Mérinos d'Arles qui transhument.

ovin/ type génétique / condition climatique / métabolisme énergétique / thermorégulation

Summary - Effects of cold, wind and rain on energy expenditure and thermoregulation of ewes from 7 genetic types. Heat production (HP) and thermoregulation of 64 ewes from 7 genetic types kept in groups of 4 animals in respiration chambers, were determined by indirect calorimetry to study the effects of the climatic conditions encountered in the Alps during spring and autumn. At an environmental temperature (Ta) of $15^{\circ} \mathrm{C}$, without wind, $H P$ of ewes averaged $14.9 \mathrm{~kJ} / \mathrm{kg} P^{0.75} / \mathrm{h}$. A reduction of Ta by $5^{\circ} \mathrm{C}$ or an increase of air speed to 2 to $4 \mathrm{~m} \cdot \mathrm{s}^{-1}$ (whirling wind) induced $9 \%$ increases in HP on average. The physical behaviour of the ewes exposed to both rain and wind at 15 or $10^{\circ} \mathrm{C}$ was altered : they stood motionless, back exposed to the wind and head bent downwards. HP increased in $1.5 \mathrm{~h}$ by 71, 101 and 120\% in the Communes des Alpes, Préalpes and Mourérous ewes, respectively. These breeds seemed to adapt rapidly to these adverse climatic conditions, as the increase in HP was only about $40 \%$ on the second day. The Merino-related breeds increased their HP more slowly (in 3 to $4 h$ ) and to a lesser extent (by 22 to 78\%). HP was not reduced on the second day, except in the Merinos d'Arles $\times$ Romanov crossbred ewes. The Merino-related breeds had a thick, dense and very insulating fleece which covered the belly and the legs. Plasma triiodothyronine, thyroxine, glucose and non-esterified fatty acids levels increased by 26, 15, 8 and 56\%, respectively, on average, after a 2-day exposure to these adverse climatic conditions. Ewes from the Préalpes, Mourérous and Communes des Alpes breeds were the most sensitive to bad weather, whereas the Merino-related genetic types seemed better adapted to bad weather, especially the ewes from the Est à Laine Merinos and Merinos d'Arles breeds which move to mountain pastures during the summer. sheep/ genetic type / climatic conditions / energy metabolism / thermoregulation

\section{INTRODUCTION}

Au cours de leur séjour en alpage, les ovins transhumants sont fréquemment exposés à des conditions climatiques défavorables (froid, intempéries) surtout au début et à la fin de la période d'estive (Dubost, 1983; Demarquet, 1985). De nombreux travaux ont porté sur l'influence de la température ambiante (Blaxter et al, 1959a, b), des précipitations (Vera et Morris, 1979), du vent seul (Joyce et al, 1966; Mc Arthur et Montheith, 1980a, b) ou du vent en association avec la pluie (Webster et Park, 1967) sur la dépense énergétique du mouton. Par ailleurs, la résistance du mouton aux intempéries (Ćhristopherson et Young, 1986) dépend également des caractéristiques de sa toison (Parer, 1963; Carter et Clarke, 1957a, b) et de son acclimatation (Slee, 1974).

La plupart des éleveurs du Sud-Est de la France pratiquent une transhumance estivale. La montée en alpages d'altitude est typique des élevages de races Mérinos 
d'Arles et Communes des Alpes. D'autres types préalpins, notamment Mourérous, seraient aussi adaptés à la transhumance. Les brebis Préalpes, a priori désavantagées par leur toison, se déplacent éventuellement en zones «intermédiaires», mais certains éleveurs les envoient également en altitude. Par ailleurs, des éleveurs ont introduit dans la région d'autres types génétiques potentiellement plus performants, (brebis des races Est à laine Mérinos, Ile de France et $\mathrm{F}_{1}$ Romanov $\times$ Mérinos d'Arles). Il était donc intéressant d'étudier les aptitudes à la transhumance des animaux de ces différents types génétiques.

Une étude a été réałisée pour déterminer l'influence de conditions climatiques bien contrôlées sur les dépenses énergétiques et les réponses physiologiques (comportement, régulation métabolique et hormonale) de brebis adultes de 7 types génétiques différents. Les animaux placés en chambres respiratoires ont été exposés successivement à l'air calme, à la pluie et au vent pendant $6 \mathrm{~h} 1 / 2$ et au vent seul, à 2 températures ambiantes $\left(15\right.$ et $\left.10^{\circ} \mathrm{C}\right)$. Ces conditions climatiques visaient à simuler les intempéries rencontrées en alpage, avec les contraintes et les limites du matériel expérimental.

\section{MATÉRIEL ET MÉTHODES}

\section{Animaux}

L'étude a porté sur des brebis âgées de 3 ans, des 7 types génétiques suivants :

- Mérinos d'Arles provenant soit du Domaine ENSAM-INRA du Merle (Salon de Provence) élevés avec utilisation de parcours et transhumance (groupe 1), soit du Domaine INRA de Fréjorgues (Montpellier) restant en permanence en bergerie (groupe 2). Les troupeaux de race Mérinos constituent l'essentiel des effectifs de moutons transhumant dans le Sud-Est.

- Est à laine Mérinos (ELM), de Lorraine, récemment introduits dans la région;

- Ile de France (I de F), du laboratoire de la production ovine (INRA Theix), ne transhumant pas;

- $F_{1}$ Romanov $\times$ Mérinos d'Arles du Domaine ENSAM-INRA du Merle, élevés avec utilisation de parcours et transhumance;

- Mourérous (M) ou Rouges, des Alpes de Haute-Provence, provenant de troupeaux transhumants;

- Communes des Alpes (CA) ou Alpines, du Valgaudemar (Haute-Provence), issues de troupeaux transhumants;

- Préalpes du Sud (P), du lycée agricole de Carméjane (Digne), qui ne transhument pas.

La race I de $\mathrm{F}$ est le résultat d'un croisement Border et Mérinos. Les 4 premiers types génétiques sont donc «mérinisés» à des degrés divers.

Chacun des 8 groupes d'animaux ( 7 types génétiques avec 2 groupes pour le type MA) était représenté par 2 lots de 4 brebis. Pour chaque type génétique, les mesures ont été effectuées 9 semaines (lot $\mathrm{A}$ ) ou 14 semaines (lot $\mathrm{B}$ ) après la tonte, ce qui a permis d'étudier l'influence du stade de toison sur la résistance aux intempéries. 


\section{Conduite des animaux}

Les mesures ont été effectuées au printemps, au cours des 2 premiers mois de gestation pendant lesquels les dépenses énergétiques des brebis ne sont pas significativement accrues. Avant la période des mesures, les animaux ont tous été logés pendant 1 à 3 mois dans un hangar fermé mais bien aéré. Ils ont reçu à volonté un bon foin de graminées, ce qui leur a permis d'être en très bon état au moment des mesures, sauf le lot $4 \mathrm{~B}$ arrivé au laboratoire juste avant les mesures. Trois jours avant et pendant la période de mesures, les brebis ont reçu une ration constituée de foin seul en quantité correspondant aux besoins d'entretien pour éliminer les effets du niveau d'alimentation. Le fourrage était distribué en un seul repas à $16 \mathrm{~h}$, après les mesures correspondant aux différents traitements climatiques.

\section{Conditions climatiques imposées}

À la suite d'essais préliminaires, les mesures ont été réalisées en chambres respiratoires dans des conditions climatiques (température, humidité relative (HR), pluie, vent) parfaitement contrôlées et selon la même séquence pour tous les types génétiques :

- lundi, de $9 \mathrm{~h}$ au mardi à $8 \mathrm{~h} \quad: 15^{\circ} \mathrm{C}, 70 \% \mathrm{HR}$, air calme;

- mardi, de 9 h à 15 h $30: 15^{\circ} \mathrm{C}, 90 \%$ HR, pluie +vent; de $15 \mathrm{~h} 30$ au mercredi à $8 \mathrm{~h}: 15^{\circ} \mathrm{C}, 70 \% \mathrm{HR}$, vent seul;

- mercredi, de $9 \mathrm{~h}$ au jeudi à $8 \mathrm{~h} \quad: 10^{\circ} \mathrm{C}, 70 \% \mathrm{HR}$, air calme;

- jeudi, de 9 h à 15 h $30 \quad: 10^{\circ} \mathrm{C}, 90 \% \mathrm{HR}$, pluie +vent; de $15 \mathrm{~h} 30$ au vendredi à $8 \mathrm{~h}: 10^{\circ} \mathrm{C}, 70 \% \mathrm{HR}$, vent seul.

Le système de climatisation des installations expérimentales n'a malheureusement pas permis d'atteindre des températures inférieures à $10^{\circ} \mathrm{C}$ avec pluie et vent sans risque de givrage de l'évaporateur. Un ventilateur puissant placé à l'avant des chambres respiratoires créait un vent turbulent compris entre 2 et $4 \mathrm{~m} / \mathrm{s}$ au niveau des animaux. Une pluie fine était simulée par 6 diffuseurs placés au plafond qui assuraient des précipitations de $30 \mathrm{~mm} / \mathrm{h}$. Les turbulences du vent projetaient la pluie sur les animaux sous différents angles.

\section{Mesures}

La production de chaleur des animaux (thermogenèse) a été déterminée en continu par calorimétrie indirecte à l'aide de 2 chambres respiratoires du type circuit-ouvert comparables à celles décrites par Vermorel et al (1973), et intégrée sur des périodes de $1 / 2 \mathrm{~h}$. Compte tenu de la place disponible dans les chambres respiratoires $(2,6 \times 1,6 \mathrm{~m})$, les mesures ont été effectuées sur des lots de 4 brebis permettant de tenir compte dans une certaine mesure d'un «effet de groupe» qui joue un rôle très important dans les conditions naturelles.

La température rectale des brebis a été déterminée (à $0,1^{\circ} \mathrm{C}$ près) à l'aide d'un thermomètre électronique, au début et à la fin de chaque traitement climatique. Les flux radiatifs de chaleur (flux absolu et flux net par rapport à une cloison en bois 
de la chambre) et les températures de surface de la toison $(T s)$ ont été mesurés à l'aide d'un fluxmètre et d'un radiothermomètre (EM 101, Linear Laboratories) en 9 régions du corps (garrot, milieu et arrière du dos, épaule, côtes, flanc, cuisse, avant et arrière du ventre) à la fin de chaque traitement climatique. $\grave{A}$ la fin de la période de mesures, un prélèvement de laine $(15 \times 6,5 \mathrm{~cm})$ a été effectué dans la partie supérieure du flanc. Les flux radiatifs absolu $(F A)$ et net $(F N)$ ainsi que la température ont été mesurés sur la laine $\left(T_{1}\right)$ et sur la partie tondue $\left(T_{2}\right)$ pour déterminer l'isolation thermique $(I T)$ de la toison dans cette région : $I T=\left(T_{2}-T_{1}\right) / F N$.

Enfin, 2 prises de sang ont été effectuées sur tous les animaux à $15 \mathrm{~h} 30$, le lundi en air calme à $15^{\circ} \mathrm{C}$ et le vendredi après $6 \mathrm{~h} 30$ d'exposition à la pluie et au vent à $10^{\circ} \mathrm{C}$, pour préciser les réponses physiologiques des animaux. Les teneurs plasmatiques en glucose et en acides gras non estérifiés $(A G N E)$ ont été déterminées par des méthodes enzymatiques et colorimétriques (Michel, 1971; Nefa C Biolyon, réf 46551$)$. Les teneurs plasmatiques en triiodothyronine $\left(T_{3}\right)$ et thyroxine $\left(T_{4}\right)$ ont été déterminées par radio-immunologie (RIA kit $\mathrm{T}_{3}$ et RIA kit $\mathrm{T}_{4}$, Amersham).

\section{Analyse statistique}

\section{Données de la thermogenèse}

Les données de thermogenèse n'ont pas été exploitées entre $16 \mathrm{~h}$ et $20 \mathrm{~h}$, en raison d'une forte augmentation après le repas. Pour prendre en compte l'effet de la digestion au cours de la nuit, l'influence du temps écoulé après le repas a été étudiée dans l'analyse de la variance de la thermogenèse sous la forme d'un facteur à 5 niveaux correspondant à 5 périodes de $4 \mathrm{~h}$ après le repas, de $20 \mathrm{~h}$ à $16 \mathrm{~h}$ le lendemain.

D'une façon générale, la thermogenèse a augmenté avec la pluie et le vent puis s'est stabilisée tout en oscillant autour d'une valeur moyenne (le plateau). C'est pourquoi, seules ont été prises en compte les valeurs à ce plateau. Une première analyse de variance de la thermogenèse a été réalisée selon le modèle suivant :

$$
\begin{gathered}
Q_{i j k l m n}=\mu+G_{i}+L_{j}+T_{k}+C_{l}+H_{m}+(G \times L)_{i j}+(G \times T)_{i k} \\
+(G \times C)_{i l}(G \times H)_{i m}+(L \times T)_{j k}+(L \times C)_{i l}+(T \times C)_{k l}+(T \times H)_{k m}+E_{i j k l m n}
\end{gathered}
$$

où $Q$ représente la thermogenèse d'un lot de 4 brebis en kcal par $\mathrm{kg}$ de poids métabolique $\left(P^{0,75}\right)$ et par h (notée en abrégé kcal*), de moyenne $\mu$ et de variance résiduelle $\sigma_{E}^{2}$,

$G_{i}=$ effet du type génétique (7 niveaux);

$L_{j}=$ effet du stade de toison (2 niveaux : 9 ou 14 semaines après la tonte);

$T_{k}=$ effet de la température de l'air (2 niveaux : 15 ou $\left.10^{\circ} \mathrm{C}\right) ;$

$C_{l}=$ effet du climat ( 3 niveaux : air calme, pluie et vent ou vent seul);

$H_{m}=$ effet du temps écoulé après le repas (5 niveaux).

Des analyses statistiques élémentaires (histogrammes sur des cellules combinant différents facteurs de variation) et l'analyse de variance selon le modèle 1 ont conduit 
pour l'interprétation des données au modèle d'analyse suivant, en combinant les facteurs ayant les plus fortes interactions entre eux :

$$
Q_{i j k l}=\mu+G L_{i}+T C_{j}+H_{k}+(G L \times T C)_{i j}+(T C \times H)_{j k}+E_{i j k l}
$$

Enfin, pour les estimations de contrastes, un modèle très proche du précédent a été utilisé :

$$
Q_{i j k}=\mu+G L T C_{i}+H_{j}+E_{i j k}
$$

Les facteurs de variation des modèles 2 et 3 sont les suivants :

$G L_{i} \quad=$ effet du facteur combiné «type génétique $\times$ stade de toison» (14 niveaux);

$T C_{j} \quad=$ effet du facteur combiné «température de l'air $\times$ climat» (6 niveaux);

$H_{j}$ ou $H_{k} \quad=$ effet du temps écoulé après le repas ( 5 niveaux);

$G L T C_{i}=$ effet de la combinaison des 2 facteurs $G L$ et $T C$ (84 niveaux);

$(G L \times T C)_{i j}=$ effet de l'interaction entre les facteurs $G L$ et $T C$ (84 niveaux);

$(T C \times H)_{j k}=$ effet de l'interaction entre les facteurs $T C$ et $H$ (20 niveaux).

Les logiciels ANVARM, VACOE et BONFER de AMANCE 81 ont été utilisés pour ces analyses et les comparisons de moyennes ajustées (Bachacou et al, 1981).

\section{Température et flux net à la surface de la toison}

Les données de température et de flux net à la surface de la toison ont été regroupées par partie du corps : le dos (garrot, milieu et arrière du dos), le côté (épaule, côtes, flanc, cuisse) et le ventre (avant et arrière) et analysées selon le modèle suivant (SAS Institute Inc, 1987) :

$$
X_{i j k l}=\mu+G_{i}+L_{j}+T_{k}+(G \times L)_{i j}+(G \times T)_{i k}+(L \times T)_{j k}+E_{i j k l}
$$

où $X$ est la variable analysée (températures et flux nets des 3 zones anatomiques) de moyenne $u$ et de variance résiduelle $\sigma_{E}^{2}$ où $G, L$ et $T$ ont été définis dans le modèle 1 .

Les données ont été analysées par partie du corps en raison d'une hétérogénéité des variances entre les mesures sur le dos et sur le ventre. La plus grande variance des valeurs obtenues sur le ventre est due au fait que les types génétiques «mérinisés» ont de la laine sous le ventre contrairement aux brebis $\mathrm{P}$ et à certains lots de CA.

\section{Paramètres sanguins}

Les concentrations plasmatiques en $\mathrm{T}_{3}, \mathrm{~T}_{4}, A G N E$ et glucose ont été analysées selon le modèle suivant :

$$
X_{i j k l}=\mu+G_{i}+C_{j}+L_{k}+(G \times C)_{i j}+(G \times L)_{i k}+(C \times L)_{j k}+E_{i j k l}
$$

où $G, C$ et $L$ ont été définis dans le modèle 1 . 


\section{Caractéristiques de la toison (densité et isolation de la laine)}

En raison d'une hétérogénéité des variances entre cellules et du faible nombre de données, la comparaison de ces paramètres entre types génétiques a été effectuée à l'aide du test $U$ de Mann et Whitney (Snedecor et Cochran, 1968) (programme STATWORKS).

\section{RÉSULTATS}

Au cours des mesures effectuées sur les brebis exposées à la pluie et au vent, de la vapeur d'eau s'est condensée en 2 occasions à la sortie de l'une des chambres respiratoires. Les mesures du débit et des concentrations en gaz carbonique et en oxygène de l'air extrait ont alors été totalement erronées. Par suite, la thermogenèse des brebis des lots $3 \mathrm{~A}$ (I de F) et 7B (P) exposées à la pluie et au vent était aberrante et n'a pas été utilisée. De plus, diverses pannes dans les installations électriques n'ont pas permis d'enregistrer les données de la thermogenèse des lots. $1 \mathrm{~B}, 2 \mathrm{~B}$ et $6 \mathrm{~B}$ en présence de vent à $15^{\circ} \mathrm{C}$.

\section{Thermogenèse des brebis}

L'analyse de variance selon le modèle 1 a mis en évidence de très fortes interactions entre le type génétique et le stade de toison d'une part, et le climat et la température ambiante d'autre part (tableau I). Ces résultats indiquent une nette différence de sensibilité entre types génétiques selon le stade de toison. L'interaction climat $x$ température ambiante s'explique par un phénomène d'adaptation détaillé plus loin.

Les résultats de l'analyse de variance selon le modèle 2 (tableau I) montrent que les facteurs qui influent le plus sur la thermogenèse sont, par ordre d'importance décroissante : le facteur combiné température - climat $(T C)$, le facteur combiné type génétique - stade de toison $(G L)$ et l'interaction $G L \times T C$. Il existe donc une différence de sensibilité entre les lots, variable selon les conditions climatiques imposées.

\section{Moyenne et variabilité de la thermogenèse}

Pour l'ensemble des conditions climatiques, la thermogenèse des brebis est en moyenne de 4,04 kcal*. La variabilité est très importante (coefficient de variation de $21 \%$ ) mais $93 \%$ de cette variance sont expliqués par le modèle 2 (tableau I).

À la température de $15^{\circ} \mathrm{C}$ en air.calme, la thermogenèse est en moyenne de $3,55 \mathrm{kcal}^{*}$ sur une période de $20 \mathrm{~h}$, soit $85 \mathrm{kcal} / \mathrm{kg} P^{0,75}$ par j. Cette valeur est proche du métabolisme à l'entretien $\left(95 \mathrm{kcal} / \mathrm{kg} P^{0,75}\right.$ d'après Tissier et Thériez, 1978).

\section{Différences entre types génétiques}

Les résultats rassemblés dans le tableau II font apparaître une différence générale entre les types Mérinos et «mérinisés» (1-4) et.les autres types génétiques (5-7). Les premiers augmentent moins que les seconds leur thermogenèse pour lutter 
Tableau I. Analyse de la variance de la thermogenèse des brebis pour l'ensemble des conditions climatiques.

\begin{tabular}{|c|c|c|c|c|}
\hline & & Modèle 1 & & Modèle 2 \\
\hline $\begin{array}{l}\text { Moyenne (kcal/kg } P^{0,75} \text { par h) } \\
\text { Coefficient de variation } \\
\text { Écart type résiduel } \\
\text { Part de la variation expliquée } p\end{array}$ & le modèle & $\begin{array}{l}4,04 \\
0,21 \\
0,298 \\
0,88\end{array}$ & & $\begin{array}{l}4,04 \\
0,21 \\
0,228 \\
0,93\end{array}$ \\
\hline $\begin{array}{l}\text { Effets principaux } \\
\text { Type génétique }(G) \\
\text { Stade de toison }(L) \\
\text { Température ambiante }(T) \\
\text { Climat }(C) \\
\text { Temps écoulé } \\
\text { après le repas }(H) \\
\text { Type génétique - Stade } \\
\text { de toison }(G L) \\
\text { Température - Climat }(T C)\end{array}$ & $\begin{array}{l}\text { F6,1572 } \\
F 1,1572 \\
F 1,1572 \\
F 2,1572 \\
\text { F4,1572 }\end{array}$ & $\begin{array}{c}246,12^{* *} \\
795,97^{* *} \\
197,03^{* *} \\
2462,91^{* *} \\
144,32^{* *}\end{array}$ & $\begin{array}{l}\text { F } 4,1481 \\
\text { F13,1481 } \\
\text { F } 5,1481\end{array}$ & $\begin{array}{r}227,42^{* *} \\
460,99^{* *} \\
1948,05^{* *}\end{array}$ \\
\hline $\begin{array}{l}\text { Interactions } \\
G \times L \\
G \times T \\
G \times C \\
G \times H \\
L \times T \\
L \times C \\
T \times C \\
T \times H \\
G L \times T C \\
T C \times H\end{array}$ & $\begin{array}{l}\text { F6,1572 } \\
\text { F6,1572 } \\
\text { F12,1572 } \\
\text { F24,1572 } \\
\text { F1,1572 } \\
\text { F2,1572 } \\
\text { F2,1572 } \\
\text { F4,1572 }\end{array}$ & $\begin{array}{r}167,55^{* *} \\
44,14^{* *} \\
58,04^{* *} \\
2,35^{* *} \\
18,46^{* *} \\
16,61^{* *} \\
205,92^{* *} \\
3,22^{* *}\end{array}$ & $\begin{array}{l}\text { F58,1481 } \\
\text { F11,1481 }\end{array}$ & $\begin{array}{r}55,55^{* *} \\
5,52^{* *}\end{array}$ \\
\hline
\end{tabular}

$* *: p<0,01$

contre les intempéries. Parmi les types du premier groupe, les brebis ELM ont une thermogenèse parmi les plus faibles, quelles que soient les conditions climatiques.

La thermogenèse du lot $\mathrm{B}$ des brebis $\mathrm{P}$, à la température de $15^{\circ} \mathrm{C}$ en air calme, semble atypique $\left(2,73 \mathrm{kcal}^{*}\right)$ comparée à celles obtenues pour le lot $\mathrm{A}\left(3,97 \mathrm{kcal}^{*}\right)$ et pour 4 lots de 4 brebis $P$ étudiés 6 ou 13 semaines après la tonte dans une expérience ultérieure $\left(4,02 \mathrm{kcal}^{*}\right)$.

Ces écarts entre types génétiques sont cependant variables (interactions significatives à $p<0,01$ ) selon les niveaux des autres facteurs de variation.

\section{Influence du temps écoulé après le repas}

Entre 4 et $8 \mathrm{~h}$ après le repas, la thermogenèse des brebis est supérieure de $6 \%$ à la moyenne de la journée $(p<0,01)$. Elle décroít ensuite progressivement pour - atteindre, entre 20 et $24 \mathrm{~h}$ après le repas, un minimum inférieur de $8 \%(p<0,01)$ à la moyenne de la journée. 


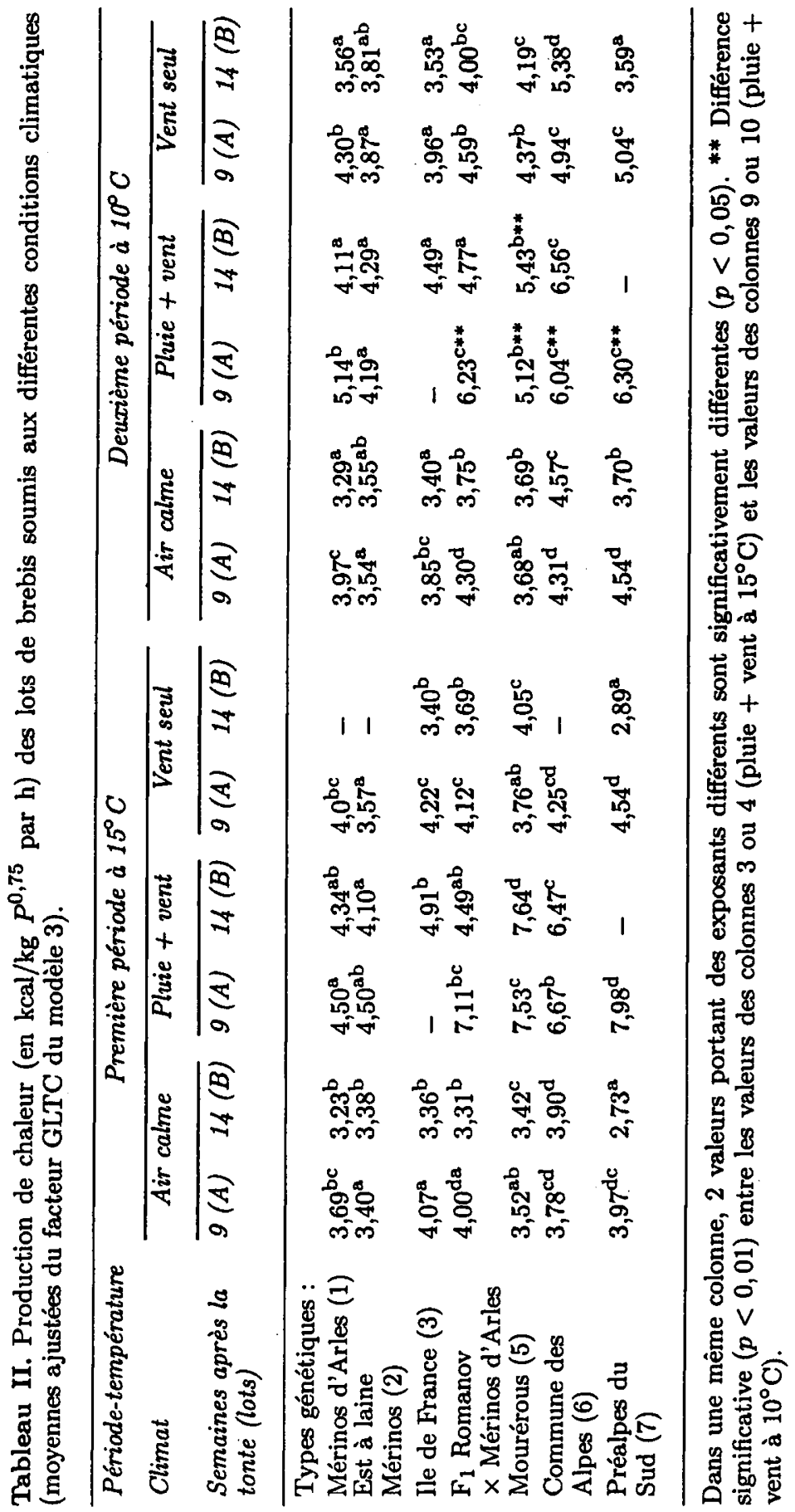


Influence de la diminution de la température ambiante de 15 à $10^{\circ} \mathrm{C}$ (tableau II)

Cet abaissement de la température ambiante entraîne, en air calme, une augmentation moyenne de $9 \%$ de la thermogenèse des brebis (de 3,55 à $\left.3,87 \mathrm{kcal}^{*}\right)(p<0,01)$. Cependant, cette augmentation de la thermogenèse est significative pour seulement 8 des 14 lots $(p<0,05): \mathrm{P}(+14,4$ à $+35,5 \%), \mathrm{CA}(+14,0$ à $+17,2 \%), \mathrm{F}_{1}(+7,5$ à $+13,3 \%)(\operatorname{lots} A$ et $B), M A(+7,6 \%, \operatorname{lot} A)$ et $M(+7,9 \%$, lot $B)$.

En présence de vent, la réduction de la température ambiante augmente la thermogenèse des brebis de types génétiques et des lots dont l'épaisseur de laine est plus faible $(p<0,01): \mathrm{CA}$ et $\mathrm{M}:+16,2 \%$ (lot $\mathrm{A}) ; \mathrm{F}_{1}:+11,4 \%$ (lot $\left.\mathrm{A}\right)$; $P:+24,2 \%(\operatorname{lot} B)$ et $+11,0 \%(\operatorname{lot} A)$.

\section{Influence du vent seul (tableau II)}

En présence de vent, la thermogenèse est augmentée de $9 \%$ en moyenne par rapport à l'air calme $(p<0,01)$ quelle que soit la température ambiante : pour les brebis CA (de $+12,4$ à $+17,7 \%$ selon les lots), $\mathrm{MA}$ (de $+8,2$ à $+8,9 \%) ; \mathrm{P}$ (de $+11,0$ à $+14,4 \%$ pour le lot $A$ seulement) et pour 2 des 4 lots $M($ de $+18,4$ à $+18,8 \%)$.

Influence de l'exposition à la pluie et au vent (tableaux II et III)

Lors de l'exposition aux intempéries, les brebis s'agitent et recherchent la position la moins défavorable, puis s'immobilisent. Les brebis $\mathrm{P}$ commencent rapidement à trembler. En revanche, les brebis ELM semblent plus à l'aise que les autres types génétiques. Le second jour, les brebis s'immobilisent plus rapidement lorsque la pluie commence à tomber et se mettent tout de suite dans la position décrire ci-dessus, ce qui semble suggérer un effet d'accoutumance aux intempéries.

Lors de la première exposition aux intempéries $\left(15^{\circ} \mathrm{C}\right.$, pluie et vent), les brebis $P$ et $M$ augmentent très rapidement leur thermogenèse et atteignent en $1,5 \mathrm{~h}$ un plateau correspondant à une augmentation de $+101 \%$ et de +114 à $+123 \%$ respectivement $(p<0,01)$ (fig 1 , tableau III). Cette forte thermogenèse permet de compenser la thermolyse puisque la température rectale des brebis est maintenue à sa valeur normale (entre 38,5 et $39,0^{\circ} \mathrm{C}$ ) à l'exception d'un animal de faible poids. Les brebis CA, proches des 2 types génétiques précédents, augmentent un peu moins rapidement (en 2,5 à $4 \mathrm{~h}$ ) et un peu moins fortement $(+66$ à $+76 \%)$ leur thermogenèse lors de la première exposition aux intempéries $(p<0,01)$ (tableau III).

Les types génétiques «mérinisés» accroissent plus lentement (en 3 à $5 \mathrm{~h}$ ) et surtout moins fortement $(\mathrm{de}+21 \grave{\mathrm{a}}+36 \%)(p<0,01)$ leur thermogenèse, excepté le lot $\mathrm{A}$ des brebis $\mathrm{F}_{1}(+78 \%)(p<0,01)$.

$\mathrm{Au}$ cours de la deuxième exposition aux intempéries $\left(10^{\circ} \mathrm{C}\right.$, pluie et vent), l'augmentation de la thermogenèse des brebis de certains types génétiques est moins importante que le premier jour : $(+39$ à $+47 \%$ au lieu de +114 à $+123 \%$ pour les brebis $\mathrm{M} ;+39 \%$ au lieu de $+101 \%$ pour les brebis $\mathrm{P} ;+40$ à $+44 \%$ au lieu de +66 à $+76 \%$ pour les brebis $\mathrm{CA} ;+45 \%$ au lieu de $+78 \%$ pour le lot $\mathrm{A}$ des brebis croisées $F_{1}(p<0,01)$ (tableau III). Ce phénomène physiologique d'accoutumance 


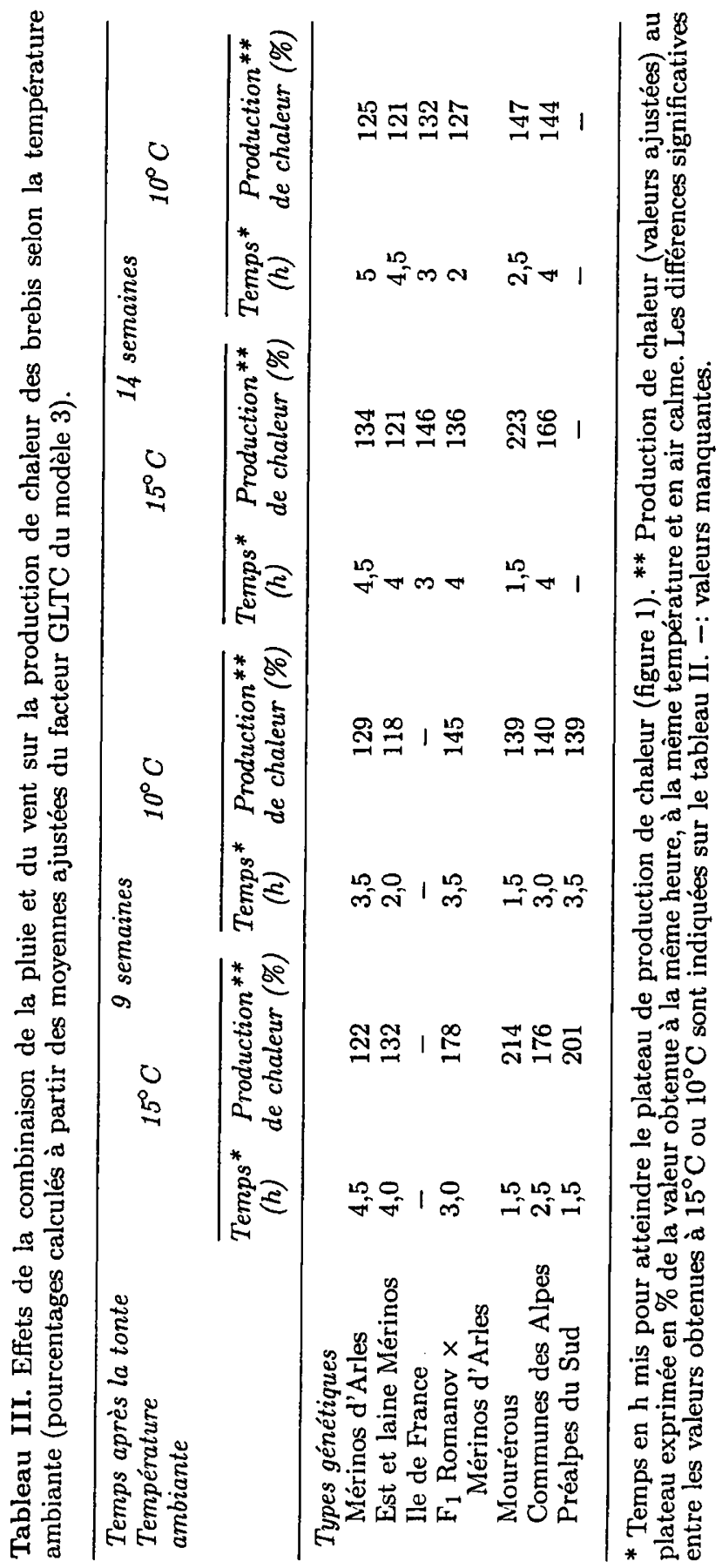




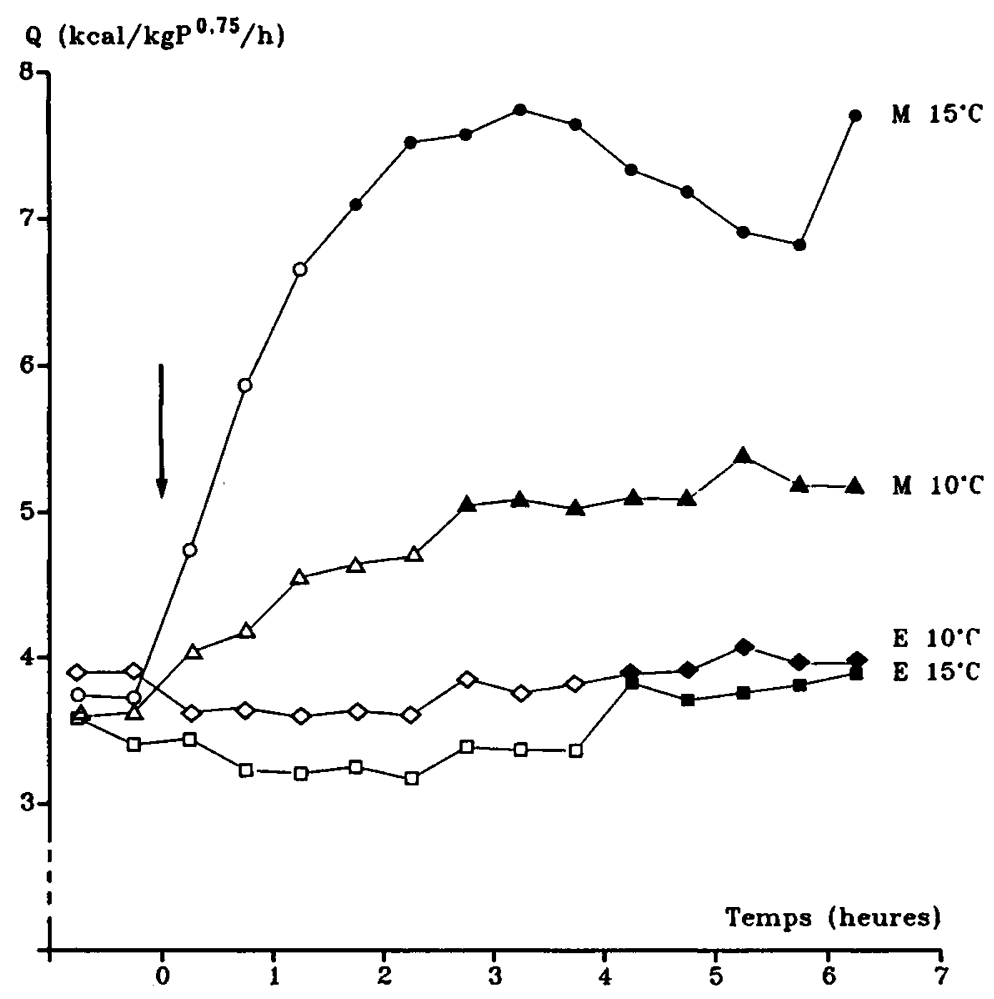

Fig 1. Évolution de la production de chaleur $(Q)$ des brebis (14 semaines après la tonte) au cours de l'exposition à la pluie et au vent (valeurs brutes). $M$ : Mourérous : $\left(O^{w}\right.$ ) $1^{\mathrm{er}}$ jour à $15^{\circ} \mathrm{C} ;(\Delta \Delta) 2^{\mathrm{e}}$ jour à $10^{\circ} \mathrm{C} ; \mathrm{E}:$ Est à laine Mérinos : (口U) $1^{\mathrm{er}}$ jour à $15^{\circ} \mathrm{C}$; $(\diamond \diamond) 2^{\mathrm{e}}$ jour à $10^{\circ} \mathrm{C}$. Les symboles pleins correspondent aux valeurs au plateau qui ont donné lieu à des analyses statistiques.

aux intempéries explique l'importance de l'interaction «température $\times$ climat» constatée dans l'analyse de variance préliminaire. De plus, il masque complètement l'augmentation éventuelle de la thermogenèse due à la réduction de la température ambiante de $15^{\circ} \mathrm{C}$ à $10^{\circ} \mathrm{C}$, que l'on observe en air calme et avec vent seul.

Les brebis MA, ELM et I de F, dont l'accroissement de la thermogenèse était modéré le premier jour d'exposition aux intempéries, ne présentent pas de phénomène d'accoutumance à la pluie et au vent.

\section{Influence du stade de toison}

La thermogenèse des brebis qui ont une toison de 9 semaines (lot $\mathrm{A}$ ) est supérieure de $11 \%$ en moyenne $(p<0,01)$ à celle des brebis qui ont une toison de 14 semaines (lot B) (tableau II). En air calme et avec le vent, la différence est la plus importante chez les brebis $\mathrm{P}\left(+41,4 \%\right.$ en moyenne), MA $(+18,6 \%)$; I de $\mathrm{F}(+17,7 \%)$ et $\mathrm{F}_{1}$ 
$(+15,5 \%)(p<0,01)$. De plus, chez ces dernières, la différence se situe entre $+30,6$ et $58,4 \%$ lors de l'exposition à la pluie et au vent $(p<0,01)$.

En revanche, les brebis ELM, CA et $M$ ne semblent pas sensibles à la différence de stade de toison. Cependant, dans le cas des brebis CA, on óbserve une très grande variabilité individuelle de la toison : la plupart des brebis du lot $6 \mathrm{~A}$ ont de la laine sous le ventre, contrairement à celles du lot $6 \mathrm{~B}$. Cette meilleure isolation compense largement l'épaisseur plus faible de la toison des brebis du lot 6A (9 au lieu de 14 semaines après la tonte).

\section{Caractéristiques des toisons}

Pour apprécier l'influence de la laine sur la perte de chaleur radiative des animaux, nous avons mesuré 3 paramètres :

- la température de surface de la toison $(T s)$, d'autant plus élevée que l'isolation thermique de l'animal est mauvaise,

- le flux net $(F N)$ qui traduit les pertes de chaleur radiatives réelles de l'animal,

- l'isolation thermique de la laine calculée comme indiqué au paragraphe Mesures.

Les 2 premiers paramètres ont été mesurés sur le dos, le côté et le ventre. Alors que les valeurs de la thermogenèse des 2 groupes de $\mathrm{MA}$ en provenance des domaines du Merle ou de Fréjorgues n'étaient pas différentes, les caractéristiques de la toison de ces 2 groupes étaient parfois distinctes au moins pour quelques paramètres, ce qui nous a amenés à distinguer ces 2 groupes dans les différentes analyses statistiques.

Valeurs moyennes et variabilité des températures et des flux radiatifs nets de surface

Les valeurs les plus élevées sont observées sur le ventre (tableau IV) en raison, d'une part, de la présence d'organes très actifs et d'une isolation tissulaire probablement plus faible et, d'autre part, d'une moindre épaisseur de la laine.

La variabilité de ces caractères est importante avec un coefficient de variation de $20 \%$, le modèle d'analyse rendant compte de plus de la moitié de la variance pour presque toutes les variables.

\section{Effet de la température ambiante (Ta) et du stade de toison}

La réduction de la température ambiante, qui est le principal facteur de variation, a des effets moins marqués sur la température de surface du ventre $(-7,7 \%)$ que sur celle du dos $(-23,5 \%)$ mieux isolé (tableau IV). L'effet du stade de toison est également significatif $(p<0,01)$. Ces 2 facteurs agissent parfois en interaction, l'effet de la baisse de la $T_{a}$ étant plus marqué chez les brebis les plus récemment tondues. 


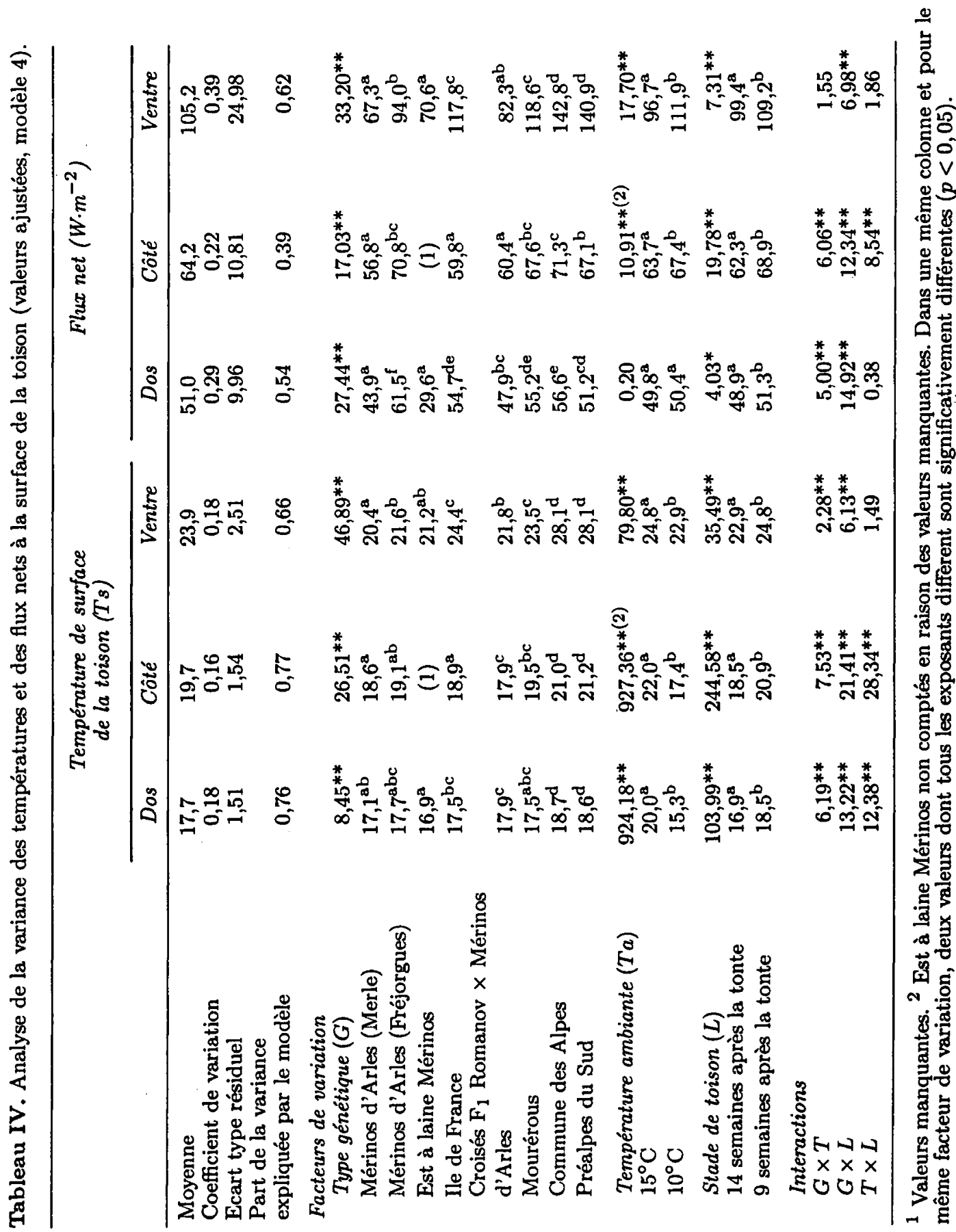


Différences entre types génétiques en relation avec les caractéristiques de la laine et de la toison

\section{Brebis à toison Mérinos ou «mérinisée»}

Les brebis MA, EML et I de F ont une toison très étendue, fermée et tassée, couvrant la tête, le ventre et les pattes. Leur laine est dense et homogène, formant des fibres fines et parallèles, ou forme des mèches carrées chez les I de F (Quittet, 1965).

Les brebis MA et EML ont les valeurs de $T s$ et $F N$ les plus basses (tableau IV), en raison d'une meilleure isolation thermique de la toison liée à une densité parmi les plus élevées (tableau V).

Les brebis I de $\mathrm{F}$ ont des pertes de chaleur radiatives $(F N)$ élevées surtout sous le ventre (tableau IV) en raison d'une isolation thermique moyenne de la toison malgré une densité élevée (tableau V).

Comparativement aux autres types génétiques de souche Mérinos, les brebis MA de Fréjorques ont une isolation thermique de la laine faible (tableau V) et des valeurs de $F N$ élevées (tableau IV) mais il peut s'agir d'un artefact dû à la composition des lots.

La toison des brebis $\mathrm{F}_{1}$ est moins étendue que celle des Mérinos et présente, selon les individus, soit des fibres droites formant de longues mèches, soit des fibres très frisées et assez courtes formant de petites mèches (toison peu isolante). La densité de la laine est élevée en raison d'une croissance rapide et son isolation thermique est moyenne (tableau V).

\section{Brebis de races alpines}

Chez les brebis de races alpines, la laine courte et peu dense forme des mèches carrées. Les brebis $M$ ont généralement de la laine sous le ventre. En revanche, il existe une grande hétérogénité dans l'étendue de la toison des CA qui sont parfois découvertes sous le ventre. Les brebis $\mathrm{P}$ sont encore moins couvertes puisque leur toison s'arrête vers le milieu du côté.

Ces particularités de l'étendue de la toison expliquent les valeurs élevées de $T s$ et $F N$ sur le ventre des brebis $\mathrm{CA}$ et $\mathrm{P}$, et dans une moindre mesure des $\mathrm{M}$. D'une façon générale, les brebis $C, M$ et surtout $P$ ont une densité de laine et une isolation thermique de la toison faibles (tableau V).

\section{Variations de quelques paramètres sanguins (tableau VI)}

En moyenne, les concentrations plasmatiques de $\mathrm{T}_{3}$ et $\mathrm{T}_{4}$ ont augmenté de $26 \%$ et $15 \%$ respectivement après $2 \mathrm{j}$ d'exposition aux intempéries $(p<0,01)$. De même, les concentrations plasmatiques de glucose et d'AGNE ont augmenté en moyenne de $8 \%(p<0,05)$ et $56 \%(p<0,01)$ respectivement dans les mêmes conditions (tableau VI).

Des différences significatives entre types génétiques existent pour $T_{3}$ et $T_{4}$ et les AGNE (tableau VI). Les brebis MA du Merle habituées à la transhumance ont les concentrations plasmatiques en $\mathrm{T}_{3}$ les plus faibles $(1,25 \mathrm{ng} / \mathrm{ml})$. Les concentrations en $\mathrm{T}_{4}$ les plus faibles sont observées pour les brebis I de $\mathrm{F}$ et $\mathrm{M}(97$ et $100 \mathrm{ng} / \mathrm{ml}$ 


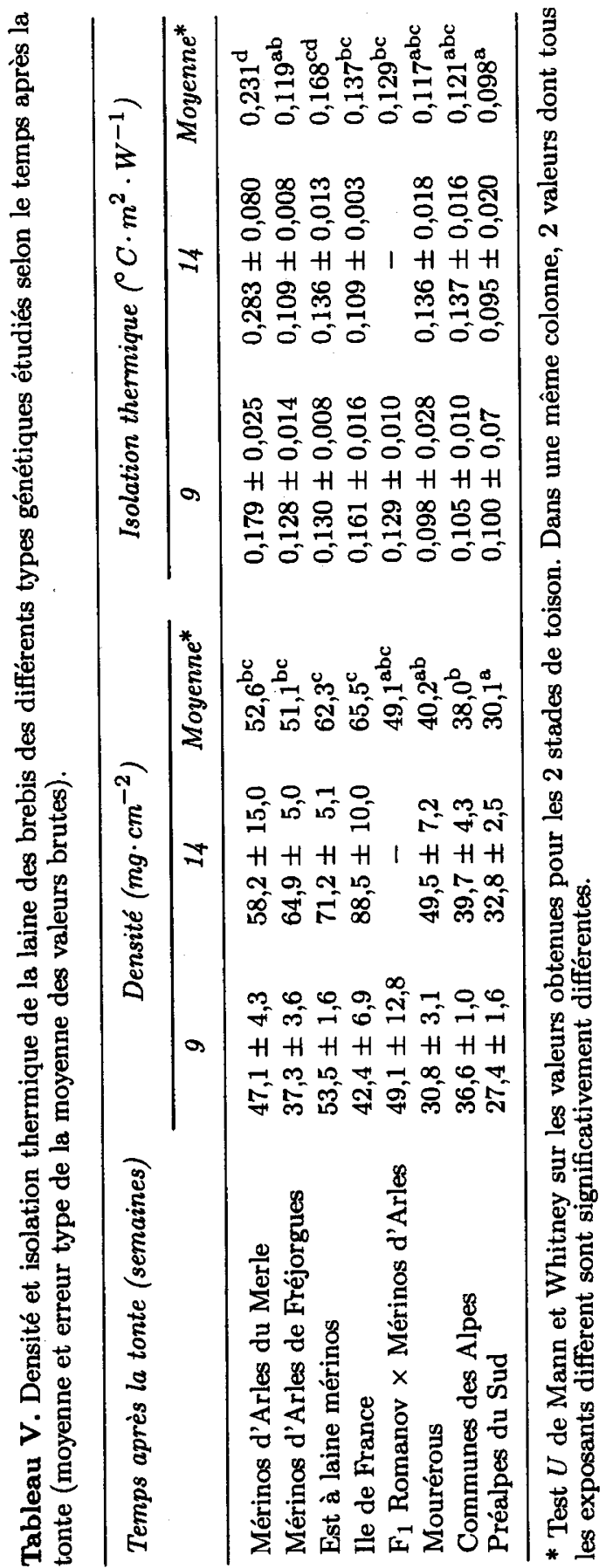


Tableau VI. Analyse de la variance des paramètres sanguins selon le modèle 5 : concentrations plasmatiques des hormones thyroïdiennes $\left(\mathrm{T}_{3}\right.$ et $\left.\mathrm{T}_{4}\right)$ du glucose et des acides gras non estérifiés (AGNE).

\begin{tabular}{|c|c|c|c|c|}
\hline $\begin{array}{l}\text { Concentration } \\
\text { plasmatique }\end{array}$ & $\begin{array}{c}T_{3} \\
(n g / m l)\end{array}$ & $\begin{array}{c}T_{4} \\
(n g / m l)\end{array}$ & $\begin{array}{l}\text { Glucose } \\
(g / l)\end{array}$ & $\begin{array}{l}A G N E \\
(m g / l)\end{array}$ \\
\hline Moyenne générale & 1,59 & 110 & 0,715 & 180,9 \\
\hline $\begin{array}{l}\text { Coefficient de } \\
\text { variation }\end{array}$ & 0,29 & 0,21 & 0,20 & 0,37 \\
\hline Écart type résiduel & 0,41 & 18,97 & 0,138 & 46,01 \\
\hline $\begin{array}{l}\text { Part de la variance } \\
\text { expliquée par le modèle }\end{array}$ & 0,23 & 0,33 & 0,10 & 0,51 \\
\hline \multicolumn{5}{|l|}{ Facteurs de variation } \\
\hline Type génétique $(G)$ & $3,03^{*}$ & $3,74^{* *}$ & 1,44 & 3,69 \\
\hline Climat $(C)$ & $26,04^{* *}$ & $20,01^{* *}$ & $5,31^{*}$ & $54,83^{* *}$ \\
\hline $\begin{array}{l}\text { - Moyenne ajustée } \\
\text { à } 15^{\circ} \mathrm{C} \text { air calme }\end{array}$ & 1,41 & 103 & 0,69 & 141 \\
\hline $\begin{array}{l}\text { - Moyènne ajustée } \\
\text { à } 10^{\circ} \mathrm{C} \text { avec pluie et vent }\end{array}$ & $\begin{array}{l}1,78 \\
(+26 \%)\end{array}$ & $\begin{array}{l}118 \\
(+15 \%)\end{array}$ & $\begin{array}{l}0,73 \\
(+8 \%)\end{array}$ & $\begin{array}{l}220 \\
(+56 \%)\end{array}$ \\
\hline Stade de toison $(L)$ & 0,55 & $7,13^{* *}$ & 0,03 & 0,75 \\
\hline Interaction $G \times C$ & 0,74 & 0,50 & 1,12 & $2,19^{*}$ \\
\hline Interaction $G \times L$ & 0,05 & $4,45^{* *}$ & 1,59 & 1,41 \\
\hline Interaction $C \times L$ & 0,71 & 0,37 & 0,02 & 0,61 \\
\hline
\end{tabular}

${ }^{*} p<0,05 ;{ }^{* *} p<0,01$.

respectivement). Les brebis MA de Fréjorgues restant toute l'année en bergerie ont les concentrations les plus fortes en $\mathrm{T}_{3}(1,84 \mathrm{ng} / \mathrm{ml})$ et $\mathrm{T}_{4}(123 \mathrm{ng} / \mathrm{ml})$.

Les concentrations en AGNE les plus faibles sont observées pour les brebis MA du Merle, ELM et CA (en moyenne $152 \pm 3 \mathrm{mg} / \mathrm{l}$ de plasma), tandis que les autres types génétiques présentent des teneurs en AGNE du plasma (198 $\pm 11 \mathrm{mg} / \mathrm{l})$ supérieures de $30 \%$ aux précédentes $(p<0,01)$.

Un effet significatif du stade de toison est observé uniquement pour $\mathrm{T}_{4}$, mais cet effet est variable selon les types génétiques : les concentrations plasmatiques de $T_{4}$ sont plus fortes 9 semaines que 14 semaines après la tonte chez les brebis MA du Merle $(+18,8 \%)$ et de Fréjorgues $(+19,4 \%), M(+22,2 \%)$, les $\mathrm{P}(+29,2)$ et les I de $\mathrm{F}(+31,3 \%)$. En revanche, elles sont plus faibles chez les brebis $\mathrm{F}_{1}(-6,9 \%), \mathrm{ELM}$ $(-10,2 \%)$ et CA $(-19,1 \%)$.

\section{DISCUSSION}

Cette étude a été réalisée dans des conditions nécessairement différentes de celles de l'Alpage pour un certain nombre d'éléments : groupes de petite taille, vent relativement faible, température minimale supérieure à celles observées en automne, exposition brutale aux intempéries. Cependant, les conditions climatiques expérimentales présentent l'avantage d'être bien contrôlées et permettent une comparaison rigoureuse des réponses des types génétiques choisis. Le dispositif expérimental 
adopté conduit également à des effectifs réduits pour les échantillons représentatifs de chaque type génétique.

L'augmentation de la thermogenèse des brebis consécutive à l'abaissement de la température de 15 à $10^{\circ} \mathrm{C}$ est en accord avec les résultats obtenus sur moutons adultes par Blaxter et al (1959a, b). Par ailleurs, les augmentations des concentrations plasmatiques de $\mathrm{T}_{3}$ et $\mathrm{T}_{4}$, de glucose et d'AGNE après $2 \mathrm{j}$ d'exposition aux intempéries, sont en accord avec les observations de Christopherson et al (1978) sur des moutons placés dans des conditions plus difficiles. Associées à l'accroissement des sécrétions de cortisol et de catécholamines observé par Thompson et al (1975), Christopherson et al (1978) et Graham et al (1981), elles permettent l'augmentation de la thermogenèse pour compenser la thermolyse accrue. En effet, ces modifications hormonales entraînent, entre autres, un accroissement de la lipolyse et une augmentation de la part de la thermogenèse provenant du catabolisme oxydatif des acides gras à chaîne longue (Sunagawa et al, 1988). L'augmentation de la sécrétion de $\mathrm{T}_{3}$ peut être très rapide et atteindre un maximum en $4 \mathrm{~h}$ (Drews et Slebodzinski, 1985 ) alors que l'augmentation de la sécrétion de $\mathrm{T}_{4}$ est plus lente (maximum en $9 \mathrm{~h}$ selon Ellis et al, 1985 ou en $24 \mathrm{~h}$ selon Drews et Slebodzinski, 1985).

L'analyse de l'ensemble des données relatives à la production de chaleur des brebis à l'aide des modèles d'analyse définis précédemment montre que les difficultés de climat expliquent l'essentiel de la variance de la thermogenèse. Ces variations peuvent être considérables, la thermogenèse des brebis des types les moins adaptés est doublée dans les conditions climatiques défavorables. Il est intéressant de souligner que le stade de toison est le facteur le plus important après le climat. Ainsi, il est probable que les différences de toison entre races (étendue, épaisseur, finesse, isolation de la laine) expliquent l'essentiel des différences de résistance aux intempéries entre types génétiques.

\section{Caractéristiques des races alpines ou préalpines}

Les brebis $\mathrm{P}$ adaptées au climat chaud et sec de la région préalpine, les brebis $\mathrm{CA}$ élevées en moyenne montagne et les brebis $M$ à vocation mixte sont handicapées dans la résistance aux intempéries par rapport aux animaux de type Mérinos. La toison moins étendue des brebis $\mathrm{CA}$ et surtout des $\mathrm{P}$ entraîne une déperdition calorique double ou triple par le ventre et le flanc. De plus, l'isolation thermique de leur laine est inférieure (de $30-40 \%$ ) à celle des brebis ELM en raison principalement d'une moindre épaisseur pour un même stade de toison (tableau V). Enfin, on observe une grande hétérogénéité entre individus d'une même race.

D'une façon générale, ces 3 types génétiques sont plus sensibles aux variations climatiques que les souches Mérinos comme le traduit l'augmentation plus forte de leur thermogenèse (tableaux II et III, fig 2) : +11 à $+35,5 \%$ pour les brebis CA et les $\mathrm{P}$, contre 0 à $+13,3 \%$ pour les Mérinos lorsque la température ambiante passe de 15 à $10^{\circ} \mathrm{C} ;+11$ à $+18,8 \%$ pour certains lots de brebis $\mathrm{CA}, \mathrm{P}$ et $\mathrm{M}$ exposées au vent, contre 0 à $+8,9 \%$ pour les types génétiques «mérinisés»; +39 à $+123 \%$ pour les brebis $\mathrm{P}, \mathrm{CA}$ et $\mathrm{M}$ contre $+18 \%$ à $+78 \%$ pour les types génétiques «mérinisés», au cours de l'exposition à la pluie et au vent.

Cependant, ces 3 types génétiques semblent capables de s'accoutumer rapidement aux intempéries puisqu'ils augmentent moins fortement leur production de chaleur 
le second jour d'exposition aux intempéries que le premier jour $(+39$ à $+47 \%$ contre +76 à $+123 \%$ ). Cette observation est particulièrement marquée pour les brebis $P$ et $M$ que l'on trouve généralement en zone préalpine, en raison d'une augmentation importante de leur thermogenèse le premier jour d'exposition aux intempéries $(+114$ à $+123 \%)$. Les brebis CA à vocation montagnarde n'augmentent leur thermogenèse que de 66 à $76 \%$ dans les mêmes conditions. Ce phénomène d'adaptation pourrait résulter d'une immobilisation plus rapide et plus complète des animaux, d'une meilleure orientation par rapport au vent, qui assure une meilleure isolation thermique (Bennet et Hutchinson, 1964), et d'une vasoconstriction plus rapide et plus efficace.

\section{Caractéristiques des races Mérinos et apparentées}

Grâce à leur toison étendue, homogène, dense, isolante, fine et à croissance plus rapide, les brebis de type Mérinos sont beaucoup plus résistantes au froid et aux intempéries que les précédentes, ce qui se traduit par une augmentation modérée de leur production de chaleur au cours de l'exposition à la pluie et au vent. Ces résultats vont donc dans le sens de l'opinion communément répandue dans la région Sud-Est d'une meilleure aptitude à la transhumance des brebis de race MA par rapport aux autres types génétiques locaux.

Les brebis I de $\mathrm{F}$ ont une production de chaleur en air calme relativement élevée mais sont peu sensibles aux variations climatiques.

Les brebis $F_{1}$ présentent 2 types de toisons aux caractéristiques très différentes : les toisons à mèches longues ont une isolation thermique comparable à celle des brebis I de F, tandis que les toisons à laine courte et bouclée sont beaucoup moins isolantes. Cette particularité de la toison mérite d'être prise en considération afin de choisir les brebis $F_{1}$ les plus résistantes pour une utilisation éventuelle de ces animaux en transhumance. Ces brebis augmentent moins fortement leur thermogenèse le second jour d'exposition à la pluie et au vent $(+27$ à $+45 \%)$ que le premier jour $(+36$ à $+78 \%)$, contrairement aux autres types génétiques de souche Mérinos.

Les brebis ELM se caractérisent par une très grande uniformité des toisons entre individus et une grande homogénéité sur le corps de l'animal avec une bonne couverture au niveau du ventre. L'isolation thermique en air calme est élevée (tableau V). De plus, la toison dense et tassée laisse difficilement pénétrer la pluie que l'on voit ruisseler sur la laine. Ces caractéristiques expliquent l'augmentation modérée $(+21$ à $+32 \%)$ de la production de chaleur lorsque les animaux sont exposés aux intempéries, sans réduction de la température rectale et sans adaptation ni modification du comportement. Sur la base de ces seuls critères, les ovins de race ELM seraient donc mieux adaptés aux intempéries que les animaux de race MA.

\section{CONCLUSION}

Cette étude à permis de préciser les caractéristiques de résistance aux intempéries de 7 types génétiques de brebis. Toutes choses égales par ailleurs, elle montre que les types génétiques de souche Mérinos (tout particulièrement ELM et MA) 
apparaissent plus aptes à résister à la pluie et au vent que les races alpines ou préalpines ( $\mathrm{P}, \mathrm{M}$ et $\mathrm{CA})$.

L'influence du stade de toison sur la thermogenèse des brebis ainsi que les caractéristiques de la laine (densité et isolation thermique) de 7 types génétiques indiquent que les différences de toison entre races (étendue, épaisseur, finesse) expliquent l'essentiel des différences de résistance aux intempéries entre les types génétiques.

La résistance au froid, au vent et à la pluie est a priori déterminante pour une bonne aptitude à la transhumance estivale en altitude. D'autres qualités d'adaptation à des situations différentes de celles étudiées dans cet article sont sans doute également nécessaires, notamment la résistance à des expositions plus longues à un froid plus rigoureux (Hocquette et al, données non publiées), ou inversement à des températures élevées avec une hygrométrie élevée simulant les périodes d'ensoleillement consécutives aux orages d'été (Hocquette et al, 1992).

On sait qu'interviennent aussi d'autres mécanismes de réponse aux agressions du climat (Christopherson et Young, 1986), tels que la variation de l'ingestion en relation avec la variation de la thermogenèse (Bocquier et al, 1988; Slee et al, 1988), l'adaptation comportementale pour la recherche d'abris (Bennet et Hutchinson, 1964), l'augmentation de la thermogenèse due au transport de l'eau contenue dans la toison mouillée (jusqu'à $+30 \%$ pour $10 \mathrm{~kg}$ d'eau d'après Gatenby et al, 1983).

Cette étude, bien que partielle, est à restituer dans une démarche de connaissance de l'adaptation aux milieux difficiles, en rapport avec une probable évolution de l'élevage ovin allaitant européen vers des systèmes d'élevages valorisateurs d'espace. Dans ce contexte, la quantification des caractères de rusticité (adaptation aux intempéries, à la marche, qualité des relations mère-agneau, homme-animal, résistance aux maladies...) devient une nécessité au même titre que celle des caractéristiques zootechniques.

Ainsi nos résultats sont déjà utilisables pour la définition des besoins alimentaires en extérieur, ou le raisonnement du choix des types génétiques en systèmes transhumants. Concernant les 2 races pures de type Mérinos (ELM et MA), les informations obtenues sur la protection assurée par la toison, incitent à une réflexion sur l'orientation de la sélection. Ces 2 races affichent en effet une vocation de souches femelles comme support de croisement terminal, avec des objectifs de sélection sur les caractéres maternels classiquement pris en compte (prolificité, désaisonnement de la reproduction, production laitière, etc); la qualité de la laine, en revanche, est pratiquement abandonnée en France depuis 2 ou 3 décennies en tant que caractère à valeur économique. Sur la base de ces résultats, on peut se demander si l'amélioration de la toison ne pourrait pas être réintroduite de façon raisonnée dans le processus global des schémas de sélection de ces races Mérinos, au moins sous l'angle de la protection vis-à-vis des intempéries.

Ces possibilités d'amélioration génétique de la rusticité par les caractéristiques de la toison, dont les coefficients d'héritabilité sont moyens à forts (Rogan, 1988), n'excluent pas celles offertes par d'autres paramètres tels que la résistance au froid des agneaux envisagés par Slee et Stott (1986) et Stott et Slee (1987) : épaisseur de la peau, isolation corporelle, persistance de l'augmentation de la thermogenèse pendant une exposition prolongée au froid. 


\section{REMERCIEMENTS}

Les auteurs remercient M-J Davicco (INRA laboratoire croissance et métabolismes des herbivores, Theix) pour sa participation à la réalisation des dosages hormonaux; $G$ Molénat (INRA, domaine du Merle) pour sa collaboration à la mise en place de l'étude, la fourniture d'animaux expérimentaux et la discussion des résultats; $M$ Thériez (INRA, laboratoire de la production ovine, Theix) pour la fourniture d'animaux expérimentaux et $\mathbf{J}$ Vernet (INRA, laboratoire croissance et métabolismes des herbivores, Theix) pour sa participation à l'analyse statistique des résultats.

Cette expérimentation portant sur des animaux de diverses origines a nécessité la participation effective et financière de nombreuses personnes et structures que nous tenons à remercier :

- ADEO - EDE des Hautes Alpes (M Bastit),

- CFPPA de Carméjane (M Demarquet),

- EDE des Alpes de Hautes Provence (MM Degeard et Mary),

- ENSA de Montpellier et domaine INRA de Fréjorgues (MM Prudhon et Gaubert),

- INERM (M Mathieu),

- SERPAM (M Mallen),

- Syndicat des éleveurs de Mourérous,

- UPRA Est à Laine Mérinos (MM Chone et Chabet)

\section{RÉFÉRENCES}

Bachacou J, Masson JP, Miller C (1981) Manuel de la programmathèque statistique AMANCE 81, CNRF-INRA 54280 Champenouy, $516 \mathrm{p}$

Bennet JW, Hutchinson JCD (1964) Thermal insulation of short lengths of Merino fleece. Aust $J$ Agric Res 15, 427-445

Blaxter KL, Mc Graham N, Wainman FW (1959a) Environmental temperature, energy metabolism and heat regulation in sheep. II. The partition of heat loss in closely clipped sheep. J Agric Sci $52,26-40$

Blaxter KL, Mc Graham N, Wainman FW (1959b) Environmental temperature, energy metabolism and heat regulation in sheep. III. The metabolism and thermal exchanges of sheep with fleece. J Agric Sci 52, 42-44

Bocquier F, Thériez M, Prache S, Brelurat A (1988) Alimentation des ovins. In : Alimentation des bovins, ovins, caprins (R Jarrige, ed) INRA, 249-279

Carter HB, Clarke WH (1957a) The hair follicle group and skin follicle population of Australian Merino sheep. Aust J Agric Res 8, 91-108

Carter HB, Clarke WH (1957b) The hair follicle of some non-Merino breeds of sheep. Aust J Agric Res 8, 109-119

Christopherson RJ, Thompson JR, Hammond VA, Hills GA (1978) Effects of thyroid status on plasma adrenaline and noradrenaline concentrations in sheep during acute and chronic cold exposure. Can J Physiol Pharm 56, 490-496 Christopherson RJ, Young BA (1986) Effect of cold environments on domestic animals. In : Grazing research at northern latitudes (O Gudmundsson, ed) Plenum Publ Corporation

Demarquet F (1985) Données météorologiques. Origine : Lycée d'Enseignement Professionnel Agricole de Carméjane (Digne) 
Drews R, Slebodzinski AB (1985) Serum principal iodothyronines and the influence of cold exposure associated with shearing in the sheep. J Thermal Biol 10, 125-129 Dubost M (1983) Utilisations des estives par les troupeaux domestiques. Stage de pastoralisme montagnard. Document ronéoté INERM CEMAGREF

Ellis T, Bradley A, Watson F, Elliott K, Smith G, McGrath M, Dolling M (1985) Protection of recently shorn sheep against adverse weather using plastic coats. Aust Vet $J$ 62, 213-218

Gatenby RM, Monteith JM, Clark JA (1983) Temperature and humidity gradient in a sheep's fleece. II The energetic significance of transients. Agric Meteorol 29, 83-101

Graham AD, Christopherson RJ, Thompson JR (1981) Endocrine and metabolic changes in sheeps associated with acclimation to constant or intermittent cold exposure. Can J Anim Sci 61, 81-90

Hocquette JF, Vermorel M, Bouix J (1992) Dépenses énergétiques et thermorégulation de 3 types génétiques de brebis exposées à des températures et hygrométries élevées. Genet Sel Evol 24 (sous presse)

Joyce JP, Blaxter KL, Park C (1966) The effect of natural outdoor environments on the energy requirements of sheep. Res Vet Sci 7, 342-359

Mc Arthur AJ, Monteith JL (1980a) Air movement and heat loss from sheep. I. Boundary layer insulation of a model sheep, with and without fleece. Proc $R$ Lond B 209, 187-208

Mc Arthur AJ, Monteith JL (1980b) Air movement and heat loss from sheep. II. Thermal insulation of fleece in wind. Proc $R$ Soc Lond B 209, 209-217

Michel MC (1971) Analyse quantitative de quelques substances azotées et glucidiques en milieu biologique. Essai de rationalisation. Thèse de Doctorat, Université de Clermont-Ferrand

Parer JT (1963) Wool length and radian heating effects in sheep. J Agric Sci 60, 141-144

Quittet E (1965) Races ovines françaises. Collection: Les races d'animaux domestiques. La Maison Rustique, Paris

Rogan IM (1988) Genetic variation and covariation in wool characteristics related to processing performance and their economic significance. In : Proceedings 3rd World Congress on Sheep and Beef Cattle Breeding. Paris, vol 2, 383-407

SAS Institute Inc (1987) SAS/STAT : Guide for personal computers. Version 6. Cary, NC, Sas Institute Inc, $1028 \mathrm{p}$

Slee J (1974) The retention of cold acclimatization in sheep. Anim Prod 19, 201-210 Slee J, Stott AW (1986) Genetic selection for cold resistance in Scottich Blackface lambs. Anim Prod 43, 396-404

Slee J, Wiener G, Wooliams C (1988) A comparison of inbred and outbred sheep on two planes of nutrition. II. Response to acute cold and heat exposure. Anim Prod 46, 221-229

Snedecor GW, Cochran WG (1968) Statistical methods, 6th edn. The Iowa Univ Press, Ames, Iowa, USA

Stott AW, Slee J (1987) The effects of litter size, sex, age, body weight, dam age, and genetic selection for cold resistance on the physiological responses to cold exposure of Scottish Blackface lambs in a progressively cooled water bath. Anim Prod 45, $477-491$ 
Sunagawa K, Murakoshi Y, Otani F, Takahashi K, Shoji Y, Tsuda T, Takahashi T (1988) Kinetics of plasma palmitic acid metabolism in fed sheep exposed to a cold environment. Jpn J Zootech Sci 59, 596-603

Thompson GE, Gardner JW, Bell AW (1975) The oxygen consumption, fatty acid and glycerol uptake of the liver in fed and fasted sheep during cold exposure. $Q J$ Exp Physiol 60, 107-121

Tissier M, Thériez M (1978) Ovins. In : Alimentation des ruminants (R Jarrige, ed) INRA, 403-448

Vera RR, Morris JG (1979) The effect of fleece length, water load and ambiant temperature on the heat production of fasting sheep. Arg Esc Vet UFMG 31, 421436

Vermorel M, Bouvier JC, Bonnet Y, Fauconneau G (1973) Construction et fonctionnement de 2 chambres respiratoires du type «circuit ouvert» pour jeunes bovins. Ann Biol Anim Biochim Biophys 13, 659-681

Webster AJF, Park C (1967) The effect of jute coats on the losses of two breeds of sheep exposed to different environments. Anim Prod 9, 483-490 